\title{
Full band quantum transport modelling with EP and NEGF methods: application to nanowire transistors
}

\author{
M. Pala \\ C2N, Université Paris-Saclay, Palaiseau, France \\ Email: marco.pala@c2n.upsaclay.fr
}

\author{
D. Esseni \\ DPIA, University of Udine, Udine, Italy \\ Email: david.esseni@uniud.it
}

\section{INTRODUCTION}

The active region of many modern electron devices consists of semiconductors structured at truly nanometric dimensions, either as ultra-thin-body FETs (UTB-FETs), or as 3D architectures such as Fin-FETs, multi-gate FETs (MuGFETs), and nanowire (NW) FETs [1]. Quantum mechanical effects have thus become prominent not only in terms of subband splitting [2], but also in terms of source-drain tunnelling in CMOS FETs [3], [4], [5], and band-to-band-tunnelling (BTBT) in Tunnel FETs (TFETs) [6], [7]. The relevance of quantum effects in nanoscale FETs is also witnessed by the fact that CMOS based quantum dots have been proposed as a platform for quantum computing [8].

The empirical tight-binding (TB) method is the most mature method for full-band quantum transport simulations based on the non-equilibrium Green's function (NEGF) formalism [9], [10], however an approach based on an Empirical Pseudopotentials (EP) Hamiltonian and a plane-waves basis has recently raised substantial interest, with contributions reported for carbon nanotubes [11], ultra-thin-body FETs [12], [13], [14], [15], and graphene nanoribbon transistors [16], [17].

We have recently reported improved methods for full band, EP based NEGF simulations of UTB-FETs [18]. In this paper we first extend the methodology to nanowires and then present complete, self-consistent simulations for nanowire Tunnel FETs with a few nanometer diameter.

\section{QUANTUM CONFINEMENT AND TRANSPORT MODELLING}

The formulation of the EP method for a bulk semiconductor has been discussed by many authors [19]. We here focus on the device structure shown in Fig. 1, namely a gate-all-around (GAA) InAs NW Tunnel-FET, and our goal is to express quantum confinement as a local operator in real space, because our previous non local formulation set a lower limit to the size of the blocks of the Hamiltonian matrix [15]. To this purpose we introduce a pseudopotential model Hamiltonian for a pseudo-oxide region, whose only purpose is to set a conduction and valence band discontinuity with respect to the semiconductor that effectively confines electrons in the semiconductor region.

To this purpose we let $V_{\mathrm{sc}}(\mathbf{r})$ and $V_{\mathrm{ox}}(\mathbf{r})$ denote the pseudopotentials describing respectively the actual semiconductor and the pseudo-oxide, and then define the overall pseudopotential $V_{1 D}(\mathbf{r})$ for the $1 \mathrm{D}$ eletron gas in the NW as

$$
V_{1 D}(\mathbf{r})=V_{\mathrm{sc}}(\mathbf{r})+V_{\mathrm{cnf}}(\mathbf{r}) \theta_{1 D}(y, z),
$$

where $V_{\text {cnf }}(\mathbf{r})$ is defined as $V_{\text {cnf }}(\mathbf{r})=\left[V_{\text {ox }}(\mathbf{r})-V_{\text {sc }}(\mathbf{r})\right]$, and $\theta_{1 D}(y, z)$ is a box function that is 1 in the oxide region and 0 in the semiconductor.

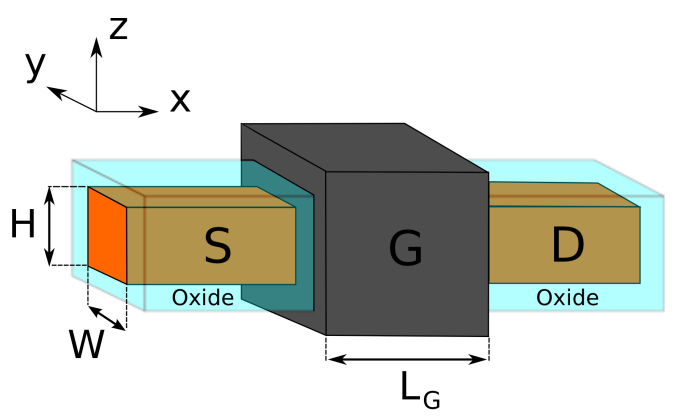

Fig. 1: Sketch of the simulated gate-all-around nanowire FET, where $x$ is the transport direction and $(y, z)$ the plane of quantum confinement.

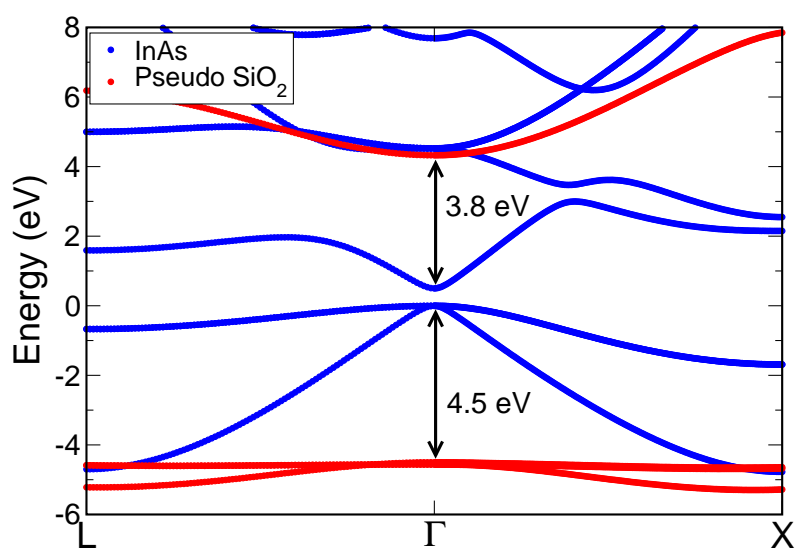

Fig. 2: Bandstructure of the pseudo- $\mathrm{SiO}_{2}$ (red) compared to that of bulk InAs (blue). The corresponding material parameters for the EP model are reported in Tab. I.

We here assumed that the pseudo-oxide has the same lattice constant $a_{0}$ as the semiconductor (hence also same reciprocal lattice), and adjusted its EP parameters in order to 


\begin{tabular}{|c|c|c|c|c|c|c|}
\hline & $U_{S 3}$ & $U_{S 8}$ & $U_{S 11}$ & $U_{A 3}$ & $U_{A 4}$ & $U_{A 11}$ \\
\hline$\psi-\mathrm{SiO}_{2}$ & -0.64 & 0.0 & 0.14 & 0.225 & 0.14 & 0.08 \\
\hline $\mathrm{InAs}$ & -0.220 & 0.0 & 0.050 & 0.080 & 0.050 & 0.030 \\
\hline
\end{tabular}

TABLE I: EP parameters (Ry) for InAs and for the pseudo$\mathrm{SiO}_{2}$.

obtain the desired values for the conduction and valence band discontinuity with respect to the semiconductor. Fig. 2 reports the energy dispersion of InAs (in blue) and of a pseudo- $\mathrm{SiO}_{2}$ material (in red), showing that the pseudo- $\mathrm{SiO}_{2}$ has a direct bandgap of about $9 \mathrm{eV}$, and that the conduction and valence band discontinuity with InAs have the desired values of about $3.8 \mathrm{eV}$ and $4.5 \mathrm{eV}$ [2].

An important feature of the $V_{1 D}(\mathbf{r})$ defined in Eq. (1) is that it is by definition local in real space, and its $\mathbf{K}$ space representation can be obtained recalling that the real space product $V_{\mathrm{cnf}}(\mathbf{r}) \theta_{1 D}(y, z)$ in Eq. (1) transforms into a convolution in reciprocal space. We here employ standard notation for wave-vectors $\mathbf{K}=\left[\left(k_{x}, \mathbf{k}_{y z}\right)+\mathbf{G}\right], \mathbf{K}^{\prime}=\left[\left(k_{x}, \mathbf{k}_{y z}^{\prime}\right)+\mathbf{G}^{\prime}\right]$, where $\mathbf{k}=\left(k_{x}, \mathbf{k}_{y z}\right)$ belongs to the reduced zone (same for semiconductor and pseudo-oxide), and $\mathbf{G}=\left(G_{x}, \mathbf{G}_{y z}\right)$ is a reciprocal lattice vector [19]. In such notation $V_{1 D}\left(\mathbf{K}-\mathbf{K}^{\prime}\right)$ is given by

$$
\begin{aligned}
& V_{\mathrm{sc}}\left(\mathbf{G}-\mathbf{G}^{\prime}\right) \delta_{\mathbf{k}, \mathbf{k}^{\prime}}+\sum_{\mathbf{G}_{y z}^{\prime \prime}} V_{\mathrm{cnf}}\left(G_{x}-G_{x}^{\prime}, \mathbf{G}_{y z}-\mathbf{G}_{y z}^{\prime}-\mathbf{G}_{y z}^{\prime \prime}\right) \\
& \quad \times \theta_{1 D}\left(\mathbf{K}_{y z}-\mathbf{K}_{y z}^{\prime}+\mathbf{G}_{y z}^{\prime \prime}\right) \delta_{k_{x}, k_{x}^{\prime}} .
\end{aligned}
$$

The energy dispersion of the $1 \mathrm{D}$ electron gas is obtained by the eigenvalues of the Hamiltonian matrix

$$
\mathbf{H}_{k_{x}}\left(\mathbf{K}, \mathbf{K}^{\prime}\right)=T(\mathbf{k}+\mathbf{G}) \delta_{\mathbf{G}, \mathbf{G}^{\prime}} \delta_{\mathbf{k}_{y z}, \mathbf{k}_{y z}^{\prime}}+V_{1 D}\left(\mathbf{K}-\mathbf{K}^{\prime}\right)
$$

where $k_{x}$ varies in the $1 \mathrm{D}$ reduced zone and $T(\mathbf{k}+\mathbf{G})$ is the well known kinetic energy term.

A real space discretization is indispensable for transport modelling with the NEGF approach, and in this work we use a simple second order, centered difference discretization of the kinetic energy operator given by [15]

$$
T(\mathbf{k}+\mathbf{G})=2 t_{0} \sum_{s=x, y, z}\left\{1-\cos \left[\left(k_{s}+G_{s}\right) d\right]\right\}
$$

where $t_{0}=\hbar^{2} / 2 m_{0} d^{2}$. In all spatial directions $s=\{x, y, z\}$ we employ the same discretization step $d=a_{0} / N_{d}$. As shown in Fig. 3, in order to attain an accetable agreement of the bandstructures obtained with a continuous and with a discretized kinetic operator, we used a large $N_{d}=30$, which also implies a larger number of Hamiltonian blocks. However, this drawback is compensated by the reduction of the size of the single block, which is the most relevant scaling parameter describing the computational burden.

The use of a second order discretization and of the local formulation of quantum confinement are the two key points that allowed us to reduce the size of the blocks of the block tridiagonal Hamiltonian matrix (compared to the formulation

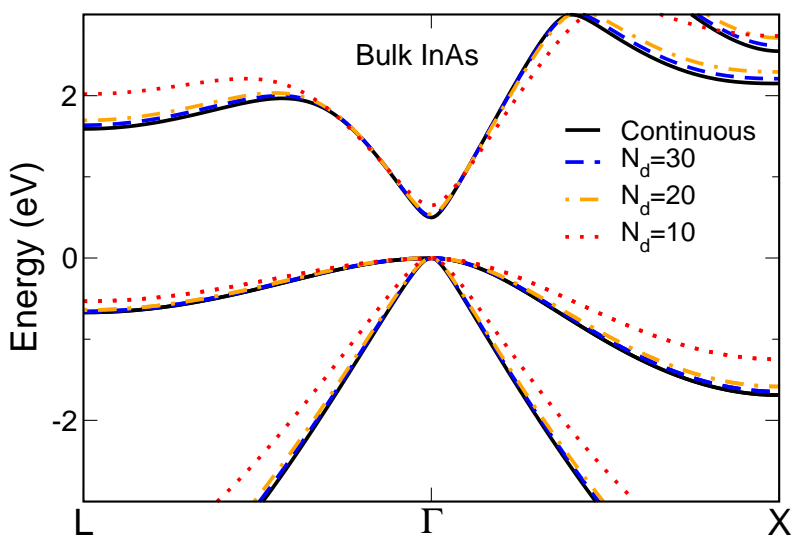

Fig. 3: Bandstructure of bulk InAs obtained with a continuous and a discretized kinetic operator with different values of $N_{d}$.

in [15]), which is a crucial parameter for NEGF based simulations. More specifically, the rank of the Hamiltonian blocks for the nanowire is $M_{1 D}=\left(2 N_{G} / N_{d}\right) N_{k_{z}} N_{k_{y}}$, where $N_{k_{y}}$ and $N_{k_{z}}$ are the number of the wavevectors in the reduced zone and are equal to $N_{c y}$ and $2 N_{c z}$, respectively, with $N_{c y}\left(N_{c z}\right)$ being the number of unit cells along $y(z)$ in the simulation domain. Hence, $M_{1 D}$ increases proportionally to the number of unit cells in the confinement directions.

A further reduction of the computational complexity can be achieved by employing a mode-space transformation [20], [21], and then by keeping only the lowest energy transverse modes, which are the most relevant for bandstructure and transport calculations in semiconductors. The mode-space Hamiltonian is obtained by means of a unitary transformation for each section of the system along $x$, namely for a single discretization point for the methodology of this work. At any discretization point $x_{l}$, the transformation matrix is equal to $\mathbf{U}^{(l)}=\left[\xi_{1}^{(l)} \cdots \xi_{N_{\text {mod }}}^{(l)}\right]$, where $\xi_{n}^{(l)}$ is the eigenvector of the eigenvalue problem

$$
\left[\mathbf{H}_{l, l}+\mathbf{H}_{l, l+1}+\mathbf{H}_{l, l+1}^{\dagger}\right] \xi_{n}^{(l)}=E_{n}^{(l)} \xi_{n}^{(l)}
$$

where $\mathbf{H}_{l, l^{\prime}}$ are the block matrices of the Hamiltonian in the hybrid basis consisting of real space in the transport direction $x$ and plane waves in the $(y, z)$ directions [15].

We found that the mode space approximation works well and it allows one to reduce significantly the size of Hamiltonian blocks for the 1D electron gas. This is not surprising because the off-diagonal blocks, $\mathbf{H}_{l, l+1}$, of the Hamiltonian matrix are diagonal matrices in the hybrid basis with a constant term $-t_{0}$ on the diagonal. In particular, the $\mathbf{H}_{l, l+1}$ blocks are independent of the transverse Bloch wave-vector $k_{z}$ (or $\left.\left(k_{y}, k_{z}\right)\right)$, so that the transverse modes $\left[\xi_{n}^{(l)}\right]$ obtained by Eq. (5) are also eigenfunctions of the diagonal blocks $\mathbf{H}_{l, l}$.

We verified that, thanks to the mode space approach, the size of the Hamiltonian blocks can be reduced to $M_{1 D}=$ $N_{\text {mod }} N_{k_{z}} N_{k_{y}}$ for a 1D gas, where, for the materials and devices analyzed in this paper, an $N_{\text {mod }}$ of about 12 is sufficient to have an agreement within a few percent between the mode 
space results and the results obtained without introducing the mode-space approximation.

In our model the charge and current density are computed in terms of the retarded, $\left[\mathbf{G}_{x \xi}\right]$, and lesser-than, $\left[\mathbf{G}_{x \xi}^{<}\right]$, Green's functions in the hybrid basis consisting of real space in the transport direction $x$ and transverse modes in the lateral directions. At a given energy $E$, Green's functions are defined as

$$
\left[\mathbf{G}_{x \xi}(E)\right]=\left[E \mathbf{I}-\left[\mathbf{H}_{x \xi}\right]-[\boldsymbol{\Sigma}(E)]\right]^{-1}
$$

and

$$
\left[\mathbf{G}_{x \xi}^{<}(E)\right]=\left[\mathbf{G}_{x \xi}(E)\right]\left[\boldsymbol{\Sigma}^{<}(E)\right]\left[\mathbf{G}_{x \xi}(E)\right]^{\dagger}
$$

where the retarded, $[\boldsymbol{\Sigma}]=\left[\boldsymbol{\Sigma}_{L}\right]+\left[\boldsymbol{\Sigma}_{R}\right]+\left[\boldsymbol{\Sigma}_{\mathrm{ph}}\right]$, and lesser-than self-energy $\left[\boldsymbol{\Sigma}^{<}\right]=\left[\boldsymbol{\Sigma}_{L}^{<}\right]+\left[\boldsymbol{\Sigma}_{R}^{<}\right]+\left[\boldsymbol{\Sigma}_{\mathrm{ph}}^{<}\right]$describe the connection to contacts (i.e. left, $L$, and right lead, $R$ ), or a possible interaction with phonons [22]. The self-energies for inelastic electron-phonon in mode-space were discussed in [23], but in this paper we do not address incoherent transport.

Moreover, because the periodicity of the unit cell is $a_{0}$, but two adjacent unit cells are connected only by the kinetic operator through the first and last discretization point, we developed a new approach to compute the contact selfenergies that, for the case at study, is more effective than the standard Sancho-Rubio algorithm [24]. More precisely, instead of computing the surface Green's function corresponding to a the entire unit cell of length $a_{0}$, as prescribed by the SanchoRubio algorithm, we focus on the surface Green's function corresponding to a single discretization point along $x$. Again, this allows us to deal with Hamiltonian blocks of reduced size and to significantly improve the computational efficiency. Details on this iterative procedure are given in Ref. [25].

The correct electrostatics of the InAs NW Tunnel-FET was simulated by self-consistently coupling the solutions of NEGF equations (6-7) with that of the 3D Poisson equation

$$
\nabla \cdot[\epsilon(\mathbf{r}) \nabla \phi(\mathbf{r})]=-e\left[p(\mathbf{r})-n(\mathbf{r})+N_{D}(\mathbf{r})-N_{A}(\mathbf{r})\right]
$$

where $\phi(\mathbf{r})$ is the electrostatic potential, $\epsilon(\mathbf{r})$ is the materialdependent permittivity, and $N_{A}(\mathbf{r}), N_{D}(\mathbf{r})$ are the acceptor and donor concentration, respectively. Before entering in the r.h.s. of Eq. (8), electron and hole concentrations were first computed on a fine discretization grid with step $d=a_{0} / N_{d}$, then, thanks to the fact that the electrostatic potential has fairly slow spatial variations on the scale of the lattice constant, they were interpolated on a coarser mesh with a discretization step $d_{c}=a_{0} / 2$.

\section{NUMERICAL RESULTS AND DISCUSSION}

The treatment of strain and arbitrary crystal orientations in our EP based model has been discussed in [18]. Fig. 4 illustrates the bandstructure for an InAs nanowire either relaxed or subject to a tensile biaxial stress and having a square cross-section with a $3.04 \mathrm{~nm}$ side. As expected the biaxial strain reduces significantly the energy gap [26], which is approximately $0.95 \mathrm{eV}$ in the unstrained system.

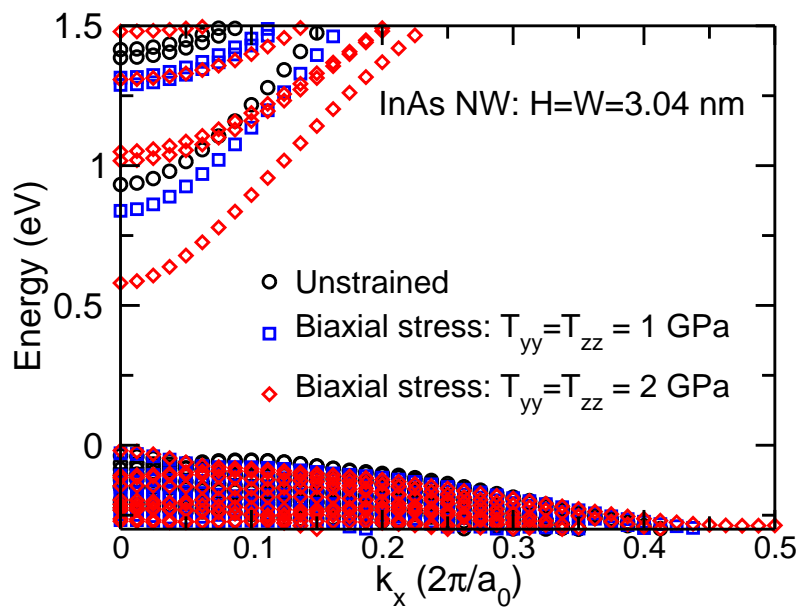

Fig. 4: Bandstructure in the 1D Brillouin zone of an InAs nanowire with different tensile biaxial stress values in the $(y, z)$ plane normal to transport dire

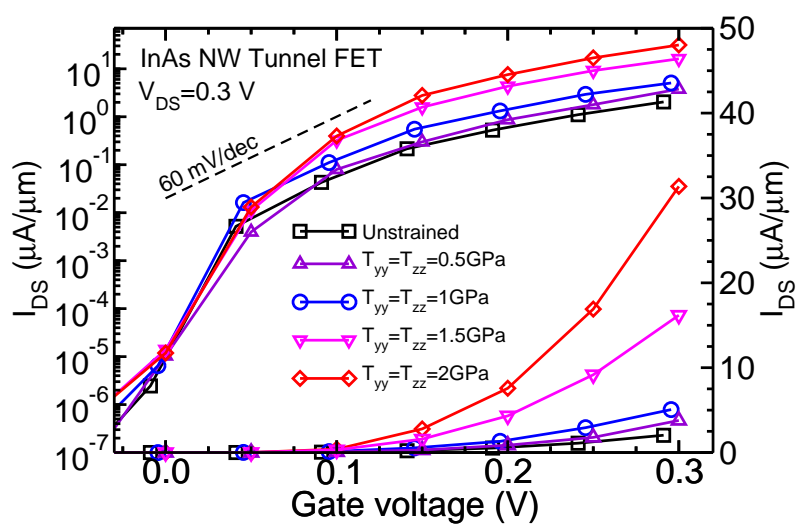

Fig. 5: Transfer characteristics of an InAs nanowire Tunnel FET either unstrained or for a tensile biaxial stress in the $(y, z)$ plane normal to the transport direction. The gate length is $L_{G}=15.2 \mathrm{~nm}$ and the cross section of the semiconductor region is a square with a $3.04 \mathrm{~nm}$ side.

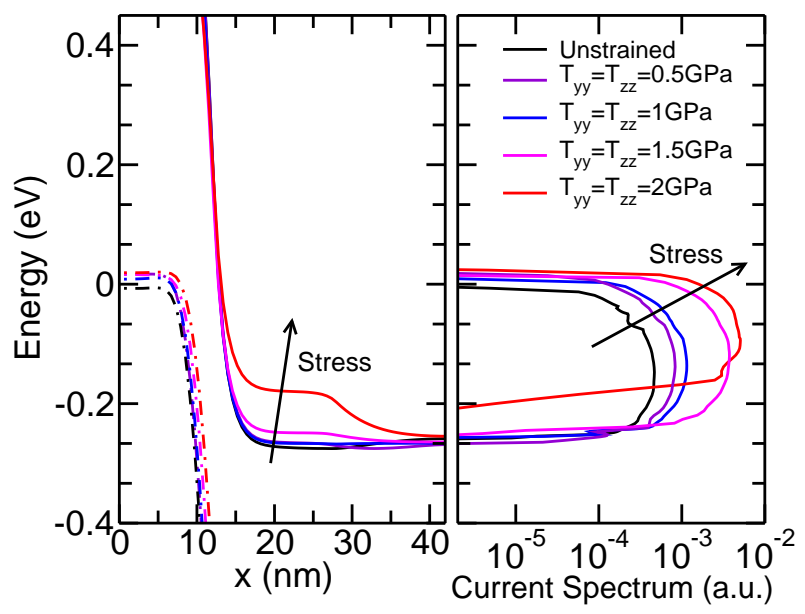

Fig. 6: Spatial profiles of the lowest conducation and highest valence subbands along the source to drain direction and current spectra for the InAs nanowire in Fig. 5 and for different stress levels. 
Fig. 5 reports the corresponding $\mathrm{I}_{D S}$ versus $\mathrm{V}_{G S}$ characteristics at $\mathrm{V}_{D S}=0.3 \mathrm{~V}$ and obtained by means of selfconsistent NEGF simulations. The metal gate workfunction was adjusted so as to have approximately the same off current $I_{O F F}=I_{D S}\left[V_{G S}=0\right]=10 \mathrm{pA} / \mu \mathrm{m}$ in all cases. As it can be seen the biaxial tensile stress improves the on state $I_{D S}$ at fixed $I_{O F F}$, without any sizeable change of the sub-threshold swing. These features are consistent with previous results obtained with a k·p Hamiltonian [26], which reinforces the confidence in the results of this paper that, for the first time, were obtained with a full band EP Hamiltonian.

Fig. 6 illustrates the subband profiles along the device channel and the current spectra for the InAs nanowire in Fig. 5 at $\mathrm{V}_{G S} \approx \mathrm{V}_{D S}=0.3 \mathrm{~V}$. As it can be seen the biaxial stress greatly increases the transmission across the channel region and consequently the on current of the Tunnel FET.

\section{CONCLUSION}

This paper has shown that the EP-NEGF methodology is a viable approach to simulate narrow NW FETs, and may thus deliver a good balance between physical accuracy and numerical burden for electron devices analysis. We argue that our transport formalism can be directly applied to plane-waves abinitio Hamiltonians, hence it may be an alternative approach to the methods based on maximally localized Wannier functions.

\section{REFERENCES}

[1] (2015) The International Technology Roadmap for Semiconductors (ITRS).

[2] D. Esseni, P. Palestri, and L. Selmi, "Nanoscale MOS Transistors - SemiClassical Transport and Applications", 1st ed. Cambridge University Press., 2011.

[3] R. Kim, U. E. Avci, and I. A. Young, "Comprehensive Performance Benchmarking of III-V and Si nMOSFETs (Gate Length $=13 \mathrm{~nm}$ ) Considering Supply Voltage and OFF-Current," IEEE Trans. on Electron Devices, vol. 62, no. 3, pp. 713-721, 2015.

[4] D. Esseni, M. Pala, and T. Rollo, "Essential Physics of the OFF-State Current in Nanoscale MOSFETs and Tunnel FETs," IEEE Trans. on Electron Devices, vol. 62, no. 9, p. 30843091, 2015.

[5] C. Grillet, D. Logoteta, A. Cresti, and M. G. Pala, "Assessment of the Electrical Performance of Short Channel InAs and Strained Si Nanowire FETs," IEEE Trans. on Electron Devices, vol. 64, no. 5, pp. 2425-2431, 2017.

[6] A. Seabaugh and Q. Zhang, "Low-Voltage Tunnel Transistors for Beyond CMOS Logic," Proceedings of the IEEE, vol. 98, no. 12, pp. 2095 -2110 , dec. 2010.

[7] D. Esseni, M. Pala, P. Palestri, C. Alper, and T. Rollo, "A review of selected topics in physics based modeling for tunnel field-effect transistors," Semiconductor Science Technology, vol. 32, p. 083005, 2017.

[8] S. D. Franceschi, L. Hutin, R. Maurand, L. Bourdet, H. Bohuslavskyi, A. Corna, D. Kotekar-Patil, S. Barraud, X. Jehl, M. S. Y.-M. Niquet, and M. Vinet, "SOI technology for quantum information processing," in IEEE International Electron Devices Meeting, pp. 339-342, 2016.

[9] M. Luisier, A. Schenk, and W. Fichtner, "Atomistic simulation of nanowires in the $\mathrm{sp} 3 \mathrm{~d} 5 \mathrm{~s}^{*}$ tight-binding formalism: From boundary conditions to strain calculations," Phys. Rev. B, vol. 74, p. 205323, 2006.

[10] G. Klimeck, S.S. Ahmed, H. Bae, N. Kharche, R. Rahman, S. Clark, B. Haley, S. Lee, M. Naumov, H. Ryu, F. Saied, M. Prada, M. Korkusinski, and T.B. Boykin, "Atomistic Simulation of Realistically Sized Nanodevices Using NEMO 3D Part I: Models and Benchmarks," IEEE Trans. on Electron Devices, vol. 54, no. 9, pp. 2079-2089, 2007.

[11] H. J. Choi and J. Ihm, "Ab initio pseudopotential method for the calculation of conductance in quantum wires," Phys. Rev. B, vol. 59, no. 3, pp. 2267-2275, 1999.
[12] Xiang-Wei Jiang, Shu-Shen Li, Jian-Bai Xia, and Lin-Wang Wang, "Quantum mechanical simulation of electronic transport in nanostructured devices by efficient self-consistent pseudopotential calculation," Journal of Applied Physics, vol. 109, p. 054503, 2011. [Online]. Available: http://dx.doi.org/doi/10.1063/1.3208067

[13] A. Garcia-Lekue, M. Vergniory, X. Jiang, and L. Wang, "Ab initio quantum transport calculations using plane waves," Progress in Surface Science, vol. 90, no. 3, pp. 292 - 318, 2015. [Online]. Available: http://www.sciencedirect.com/science/article/pii/S0079681615000209

[14] M. Pala, O. Badami, and D. Esseni, "NEGF based transport modeling with a full-band, pseudopotential Hamiltonian: Theory, Implementation and Full Device Simulations," in IEEE International Electron Devices Meeting, pp. 35.1.1-35.1.4, 2017.

[15] M. G. Pala and D. Esseni, "Full-band quantum simulation of electron devices with the pseudopotential method: Theory, implementation, and applications," Phys. Rev. B, vol. 97, p. 125310, Mar 2018. [Online]. Available: https://link.aps.org/doi/10.1103/PhysRevB.97.125310

[16] J. Fang, W. G. Vandenberghe, B. Fu, and M. V. Fischetti, "Pseudopotential-based electron quantum transport: Theoretical formulation and application to nanometer-scale silicon nanowire transistors," Journal of Applied Physics, vol. 119, p. 035701, 2016. [Online]. Available: http://dx.doi.org/doi/10.1063/1.3208067

[17] J. Fang, S. Chen, W. G. Vandenberghe, B. Fu, and M. V. Fischetti, "Theoretical Study of Ballistic Transport in Silicon Nanowire and Graphene Nanoribbon Field-Effect Transistors Using Empirical Pseudopotentials," Electron Devices, IEEE Transactions on, vol. 64, no. 6, pp. 2758 - 2764, 2017.

[18] M. Pala, O. Badami, and D. Esseni, "Transport models based on NEGF and empirical pseudopotentials: a computationally viable method for self-consistent simulation of nanoscale devices," in IEEE International Electron Devices Meeting, pp. 33.1.1-33.1.4, 2018.

[19] M.L. Cohen and J.R. Chelikowsky, Electron Structure and Optical Properties of Semiconductors. Springer Series in Solid-State Sciences. Springer-Verlag Berlin Heidelberg New York London Tokyo, 1988.

[20] R. Venugopal, Z. Ren, S. Datta, and M. Lundstrom, "Simulating quantum transport in nanoscale transistors: Real versus mode-space approaches," J. Appl. Phys., vol. 92, no. 7, pp. 3730-3739, 2002.

[21] S. Poli, M. G. Pala, T. Poiroux, S. Deleonibus, and G. Baccarani, "Size Dependence of Surface-Roughness-Limited Mobility in SiliconNanowire FETs," IEEE Transactions on Electron Devices, vol. 55, no. 11, pp. 2968-2976, Nov 2008.

[22] G.D. Mahan, Many-Particle Physics. New York: Plenum Press, 1988.

[23] K. Rogdakis, S. , E. Bano, K. Zekentes, and M. Pala, "Phonon-and surface-roughness-limited mobility of gate-all-around $3 \mathrm{C}-\mathrm{SiC}$ and $\mathrm{Si}$ nanowire FETs," Nanotechnology, vol. 20, no. 29, p. 295202, 2009.

[24] M. P. L. Sancho, J. M. L. Sancho, and J. Rubio, "Quick iterative scheme for the calculation of transfer matrices: application to Mo (100)," Journal of Physics F: Metal Physics, vol. 14, no. 5, p. 1205, 1984. [Online]. Available: http://stacks.iop.org/0305-4608/14/i=5/a=016

[25] M. G. Pala and D. Esseni, "Quantum transport models based on NEGF and empirical pseudopotentials for accurate modelling of nanoscale electron devices," to be published, 2019.

[26] F. Conzatti, M. Pala, D. Esseni, E. Bano, and L. Selmi, "Strain-Induced Performance Improvements in InAs Nanowire Tunnel FETs," Electron Devices, IEEE Transactions on, vol. 59, no. 8, pp. 2085-2092, 2012. 